\title{
EFEITO DA ADIÇÃO DE GELATINA EM EMULSÕES PREPARADAS COM QUITOSANA PARA ENCAPSULAR CONCENTRADOS DE ÁCIDOS GRAXOS INSATURADOS DE ÓLEO DE PESCADO
}

\author{
D. L. BOTELHO ${ }^{1}$, P. P. SILVA ${ }^{1}$, V. M. ESQUERDO ${ }^{1}$ e L. A. A. PINTO ${ }^{1}$ \\ ${ }^{1}$ Universidade Federal do Rio Grande, Escola de Química e Alimentos \\ E-mail para contato: nessafurg@gmail.com
}

\begin{abstract}
RESUMO - O óleo de pescado é uma fonte rica em ácidos graxos insaturados, porém sua utilização no preparo de produtos alimentícios é tarefa difícil devido a sua baixa solubilidade em água e instabilidade oxidativa. A formação de emulsões com polímeros como quitosana e gelatina pode facilitar sua incorporação em alimentos. O trabalho objetivou utilizar os polímeros quitosana e gelatina para avaliar a capacidade de formar de emulsões estáveis no encapsulamento de concentrados de ácidos graxos insaturados (AGI) obtidos de óleo de vísceras de carpa (Cyprinus carpio). Foram testadas as estabilidades de emulsões contendo os concentrados de AGI com os materiais de parede $(2 \% \mathrm{~m} / \mathrm{v})$ : quitosana pura e misturas quitosana:gelatina de 75\%:25\% e 50\%:50\%. As emulsões estáveis foram avaliadas quanto a potencial Zeta, distribuição de tamanho e índice de polidispersão. A mistura contendo $50 \%$ de cada polímero não foi capaz de formar emulsão. As emulsões com quitosana pura e com 75\%:25\% quitosana:gelatina ficaram estáveis durante 30 dias, apresentaram potencial Zeta positivo e índice de polidispersão abaixo de 0,3 . Além disso, a adição de pequena quantidade de gelatina nas emulsões (25\%) proporcionou uma diminuição no tamanho da partícula (de $480 \mathrm{~nm}$ para $156 \mathrm{~nm}$ ) e na turbidez, demonstrando que é importante o estudo da quantidade de gelatina adicionada para a elaboração de emulsões estáveis.
\end{abstract}

\section{INTRODUÇÃO}

Existe um crescente interesse das indústrias de alimentos por incorporar compostos bioativos em suas formulações a fim de desenvolver alimentos funcionais e nutracêuticos (Lee e McClements, 2010). As emulsões são sistemas coloidais que consistem em duas fases imiscíveis, que podem ser de óleo em água ou água em óleo. Alimentos, pesticidas e fármacos têm utilizado emulsões para encapsular, proteger e distribuir componentes lipofílicos, como por exemplo, o óleo de pescado (Karthik e Anandharamakrishnan, 2016).

Entre os numerosos compostos bioativos a que se associa um efeito saudável quando incorporados na dieta destacam-se os ácidos graxos insaturados de cadeia longa, particularmente os da série ômega 3. O óleo de pescado constitui uma fonte natural de ácidos graxos insaturados (Karthik e Anandharamakrishnan, 2016; Shahidi, 2015). No entanto, a produção de alimentos enriquecidos com ácidos graxos insaturados (AGI) é tarefa difícil, 
devido à sua baixa solubilidade em água e susceptibilidade a oxidação (Augustin e Sanguansri, 2015). Nesse sentido a encapsulação por formação de emulsões serve como uma alternativa tecnológica que permite resolver esses problemas.

A quitosana, um polímero catiônico derivado de recursos naturais renováveis, vem sendo estudada como material de parede devido as suas características, como biodegradabilidade e propriedades formadoras de filme (Contri et al., 2014). A gelatina é uma proteína derivada da hidrólise parcial do colágeno animal, e possui grande número de diferentes grupos funcionais acessíveis na sua estrutura. Seu uso em alimentos se destaca devido ao seu alto teor de aminoácidos como a glicina, prolina e alanina, agregando valor nutritivo ao alimento por ser totalmente digerível. A adição de gelatina na formação de emulsões pode melhorar fluidez de emulsões, e prevenir coalescência e separação de fases em diferentes sistemas de emulsão em que estão dispersos óleos e glóbulos de gorduras (Duconseille et al., 2015; Sahoo et al., 2015). Por apresentar as vantagens mencionadas, o presente trabalho teve como objetivo avaliar a viabilidade da substituição parcial de quitosana por gelatina na formação de emulsões estáveis para encapsular concentrados de ácidos graxos insaturados obtidos de óleo de vísceras de carpa comum.

\section{MATERIAL E MÉTODOS}

\subsection{MATERIAIS DE PAREDE}

Quitosana: a quitosana (grau de desacetilação de $86 \pm 1 \%$ e massa molar de $153 \pm 2$ $\mathrm{kDa}$ ) foi produzida a partir de resíduos de camarão (Penaeus brasiliensis) (Weska et al., 2007). A quitina foi isolada através das etapas de desmineralização, desproteinização e desodorização/despigmentação. Após foi realizada reação de desacetilação da quitina, obtendo-se ao final do processo a quitosana. A quitosana foi purificada e seca em leito de jorro até umidade comercial (10,0\%, em base úmida) (Dotto et al., 2011).

Gelatina: A gelatina de peles de suíno de grau alimentício/farmacêutico (Tipo A) com força do gel de $308 \pm 5 \mathrm{~g}$ e ponto de fusão de $28,5 \pm 0,5{ }^{\circ} \mathrm{C}$ foi adquirida da Sigma-Aldrich (Merck, São Paulo, Brasil).

\subsection{CONCENTRADOS DE AGI}

O óleo bruto foi extraído de vísceras de carpa comum (Cyprinus carpio) por método termomecânico. O óleo bruto passou pelas etapas do processo de refino químico (degomagem, neutralização, lavagem, secagem e branqueamento). O óleo branqueado foi submetido à reação de hidrólise química com $\mathrm{KOH}$ alcoólico como catalisador, obtendo-se os ácidos graxos livres, os quais foram misturados a uma solução alcoólica de ureia para complexação dos ácidos graxos saturados e obtenção da fração concentrada de AGI (Crexi et $a l ., 2012)$. Foi calculado o índice de peróxido (Cd 8-53) dos concentrados de AGI (AOCS 1980). A análise do perfil de ácidos graxos foi realizada por cromatografia gasosa (Varian3400 CX, Palo Alto, USA) (Metcalfe et al., 1966). 


\subsection{PREPARO E CARACTERIZAÇÃO DAS EMULSÕES DE CONCENTRADOS DE AGI COM POLÍMEROS}

O método de emulsão óleo-água foi usado para obter as suspensões. Inicialmente foram preparadas duas fases. Na fase aquosa, foram preparadas soluções de concentração $2 \%(\mathrm{~m} / \mathrm{v})$ contendo os biopolímeros. Foram elaboradas uma solução com quitosana pura (como controle) e duas soluções com substituições de $25 \%$ e $50 \%(\mathrm{~m} / \mathrm{m})$ de quitosana por gelatina, respectivamente. As soluções aquosas obtidas foram agitadas (300 rpm) em agitador magnético à temperatura ambiente. Na fase oleosa, os concentrados de AGI (proporção AGI:material de parede de 1:2) e o tensoativo Tween $80(5 \% \mathrm{~m} / \mathrm{m}$ em relação ao material de parede) foram vertidos na fase aquosa. Após, as soluções foram homogeneizadas em agitador mecânico (Dremel, 1100-01, São Paulo, Brasil) a 10.000 rpm por 10 min.

A estabilidade das emulsões foi determinada segundo Klaypradit e Huang (2008), em tubos de ensaio durante 30 dias. A análise do potencial Zeta foi utilizada para refletir o potencial de superfície das partículas (Ahmad et al., 2011). A distribuição de tamanho e índice de polidispersão foram determinadas utilizando o método de espalhamento de luz dinâmico (Zetasizer Nano ZS90, Malvern Instruments, UK) (Ahmad et al., 2011). A turbidez foi determinada usando espectrofotômetro (Biospectro, SP-22, Curitiba, Brasil).

\section{RESULTADOS E DISCUSSÃO}

\subsection{CARACTERIZAÇÃO DOS CONCENTRADOS DE AGI}

A Tabela 1 apresenta a soma dos ácidos graxos presentes nos concentrados de AGI. A fração não complexada, contendo os concentrados, apresentou um teor total de cerca de 88,2\% de AGI. Crexi et al. (2012) encontraram cerca de 68\% de AGI em óleo de carpa refinado, mostrando que o processo de concentração de óleo pela complexação de ureia foi efetivo.

Tabela 1 - Soma dos ácidos graxos presentes nos concentrados de AGI obtidos de vísceras de carpa (Cyprinus carpio).

\begin{tabular}{|c|c|}
\hline Ácidos graxos & Concentrados de AGI (\%)* \\
\hline$\sum$ AGS (\%) & $7,5 \pm 0,3$ \\
\hline$\sum$ AGMI (\%) & $38,2 \pm 0,1$ \\
\hline$\sum$ AGPI (\%) & $50,0 \pm 0,2$ \\
\hline$\sum$ AGni (\%) & $4,3 \pm 0,2$ \\
\hline
\end{tabular}

Os ácidos graxos da série omega-3 (ácido $\alpha$-linolênico (C18: 3), ácido eicosatrienóico (C20: 3), ácido eicosapentaenóico-EPA (C20: 5), ácido docosahexaenóico-DHA (C22: 6) foram aproximadamente 54\% do total, e o ácido linoléico (C18: 2 - $\omega 6)$, importante na síntese de ácido araquidônico, apresentou aproximadamente $16 \%$ do total de ácidos graxos, sendo então os concentrados uma rica fonte de ácidos graxos essenciais. $\mathrm{O}$ valor de peróxido dos 
concentrados de AGI foi de 3,6 meq $\mathrm{kg}^{-1}$, estando dentro dos padrões de qualidade desejados (ANVISA, 1999).

\subsection{CARACTERIZAÇÃO DAS EMULSÕES}

Como pode ser visto na Figura 1, após a homogeneização as emulsões formadas de quitosana pura (Fig. 1(a)) e proporção de quitosana:gelatina 75\%:25\% (Figuras 1(b)) apresentaram-se estáveis após 30 dias de armazenamento. Porém, a suspensão com substituição de $50 \%$ de gelatina não foi capaz de formar emulsão, apresentando separação de fases (Figura 1 (c)). Sabe-se que a gelatina tem sido um tensoativo eficiente, capaz de atuar como emulsionante em emulsões de óleo em água. No entanto, quando usado em grande proporção pode produzir gotas relativamente grandes, prejudicando a estabilidade (Piorkowski e McClements, 2014).

Figura 1 - Emulsões formadas utilizando como material de parede: (a) 100\% quitosana, (b) $75 \%: 25 \%$ quitosana:gelatina e (c) 50\%:50\% quitosana -gelatina.

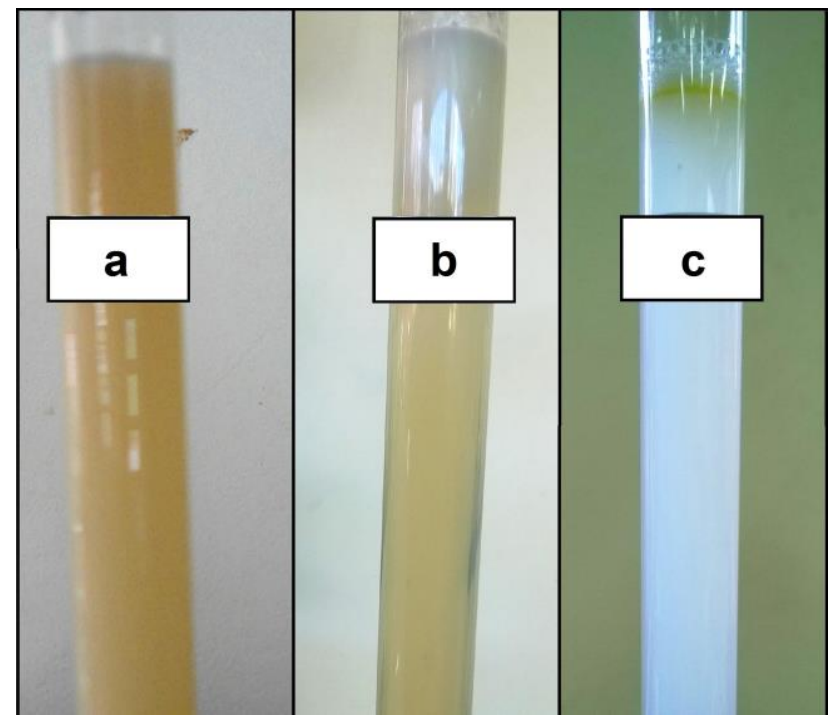

A Tabela 2 apresenta os valores de potencial Zeta, distribuição de tamanho e índice de polidispersão das partículas presentes nas emulsões formadas. Os valores de potencial Zeta foram positivos com carga interfacial de 26,4 e $31,2 \mathrm{mV}$ para a emulsão com quitosana pura e a adicionada de $25 \%$ de gelatina, respectivamente. Os resultados positivos podem ser atribuídos à presença de quitosana, que apresenta uma carga positiva na molécula quando em um meio ácido. Os valores de potencial Zeta acima de $20 \mathrm{mV}$ sugerem emulsões estáveis, devido à repulsão entre as partículas e impedem a agregação (Piorkowski e McClements, 2014). A diminuição da carga de gotículas nas misturas pode ser explicada pela substituição parcial de quitosana (biopolímero catiônico), para a gelatina que é um biopolímero anfotérico e, portanto, não causa carga na superfície das gotas.

A substituição de $25 \%$ de quitosana por gelatina proporcionou uma diminuição significativa no tamanho das partículas (de $480 \mathrm{~nm}$ para $156 \mathrm{~nm}$ ) (Tabela 2). Menores tamanhos de partículas proporcionam a formação de emulsões mais estáveis, com menor turbidez (Tabela 2) e maior transparência. Por outro lado, a taxa de oxidação dos lipídeos 
aumenta à medida que o tamanho da partícula diminui, devido à maior área de superfície exposta à fase aquosa (Osborn e Akoh, 2004). As amostras apresentaram índice de polidispersão menor que 0,3 (Tabela 2), com distribuição monomodal, indicando homogeneidade, com as gotículas distribuindo-se em uma faixa estreita.

Tabela 2 - Valores de potencial Zeta, distribuição de tamanho e índice de polidispersão para as emulsões formadas.

\begin{tabular}{|c|c|c|c|c|}
\hline $\begin{array}{l}\text { Material de } \\
\text { Parede }\end{array}$ & 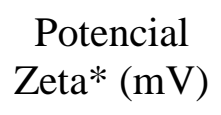 & $\begin{array}{l}\text { Distribuição de } \\
\text { tamanho* (nm) }\end{array}$ & $\begin{array}{c}\text { Índice de } \\
\text { polidispersão* }\end{array}$ & Turbidez* \\
\hline quitosana pura & $30,2 \pm 0,3^{\mathrm{a}}$ & $480,2 \pm 7.1^{\mathrm{a}}$ & $0,263 \pm 0,003^{\mathrm{a}}$ & $0.542 \pm 0.003^{\mathrm{a}}$ \\
\hline $\begin{array}{c}75 \%: 25 \% \\
\text { quitosana:gelatina }\end{array}$ & $26,4 \pm 0,8^{\mathrm{b}}$ & $156,8 \pm 3,4^{b}$ & $0,279 \pm 0,024^{\mathrm{a}}$ & $0.473 \pm 0.005^{b}$ \\
\hline
\end{tabular}

\section{CONCLUSÃO}

Foram preparadas emulsões de concentrados de ácidos graxos insaturados (AGI) adicionados dos polímeros quitosana na forma pura e substituída parcialmente por gelatina. A substituição de $50 \%$ de gelatina impossibilitou a formação de emulsão. As emulsões com quitosana pura e 75\%:25\% de quitosana:gelatina foram estáveis durante 30 dias, apresentaram potencial Zeta positivo e índice de polidispersão abaixo de 0,3 . A substituição de $25 \%$ de quitosana por gelatina nas emulsões provocou diminuição no tamanho da partícula (de 480 $\mathrm{nm}$ para $156 \mathrm{~nm}$ ) e na turbidez. Estes resultados demonstram que a proporção dos polissacarídeos na composição das emulsões tem um papel importante para a formação e utilização das emulsões.

\section{REFERENCIAS}

AHMAD, J.; KOHLI, K.; MIR, S. R.; AMIN S. Formulation of self-nanoemulsifying drug delivery system for telmisartan with improved dissolution and oral bioavailability. $J$. Dispersion Sci. Technol., v. 32, p. 958-968, 2011.

ANVISA. Diário Oficial da República Federativa do Brasil, 1999, 82.

AOCS. American Oil Chemists' Society. Official and Tentative Methods of American Oil Chemists' Society, 3rd edn. USA, Champaig, 1980.

AUGUSTIN, M. A.; SANGUANSRI, L. Challenges and solutions to incorporation of nutraceuticals in foods. In: Doyle M. P.; Klaenhammer T. R. (Eds.). Ann. Rev. Food Sci. Technol., v. 6, p. 463-477, 2015.

CONTRI, R. V.; SOARES, R. M. D.; POHLMANN, A. R.; GUTERRES S. S. Structural analysis of chitosan hydrogels containing polymeric nanocapsules. Mater. Sci. Eng. C, v. 42, p. 234-242, 2014. 
CREXI, V. T.; MONTE, M. L.; MONTE, M. L.; PINTO, L. A. A. Polyunsaturated fatty acid concentrates of carp oil: chemical hydrolysis and urea complexation. J. Am. Oil Chem. Soc., v. 89, p. 329-334, 2012.

DOTTO, G. L.; SOUZA, V. C.; PINTO, L. A. A. Drying of chitosan in a spouted bed: The influences of temperature and equipment geometry in powder quality. LWT-Food Sci. Technol., v. 44, p. 1786-1792, 2011.

DUCONSEILlE, A.; ASTRUC, T.; QUINTANA, N.; MEERSMAN, F.; SANTELHOUTELLIER, V. Gelatin structure and composition linked to hard capsule dissolution: A review. Food Hydrocolloid., v. 43, p. 360-376, 2015.

ESQUERDO, V. M.; DOTTO, G. L.; PINTO, L. A. A. Nanoemulsions containing Unsaturated fatty acid Concentrates. In: Grumezescu, A. M. (Eds.), Emulsions: Nanotechnology in the Agri-Food Industry (pp.71-100), Cap. 3, Ed. Elsevier. 2016

KARTHIK, P.; ANANDHARAMAKRISHNAN, C. Enhancing omega-3 fatty acids nanoemulsion stability and in-vitro digestibility through emulsifiers. J. Food Eng., v. 187, p. 92-105, 2016.

KLAYPRADIT, W.; HUANG, Y. W. Fish oil encapsulation with chitosan using ultrasonic atomizer. LWT-Food Sci. Technol., v. 41, p. 1133-1139, 2008.

LEE, S.J.; MCCLEMENTS, D.J. Fabrication of protein-stabilized nanoemulsions using a combined homogenization and amphiphilic solvent dissolution/evaporation approach. Food Hydrocolloids, v. 24, p. 560-569, 2010.

METCALFE, L. D.; SCHIMITZ, A. A. PELKA J. R. Rapid preparation of fatty acid esters from lipids for gas liquid chromatography. Anal. Chem., v. 38, p. 510, 1966.

OSBORN, H. T.; AKOH, C. C. Effect of emulsifier type, droplet size, and oil concentration on lipid oxidation in structured lipid-based oil-in-water emulsions. Food Chem., v. 84, p. 451-456, 2004

PIORKOWSKI, D. T.; MCCLEMENTS, D. J. Beverage emulsions: Recent developments in formulation, production, and applications. Food Hydrocolloids, v. 42, p. 5-41, 2014

QADIR, A.; FAIYAZUDDIN, M. D.; HUSSAIN, M. D. T.; ALSHAMMARI, T. M.; SHAKEEL, F. Critical steps and energetics involved in a successful development of a stable nanoemulsion. J. Mol. Liq., v. 214, p. 7-18, 2016

SAHOO, N.; SAHOO, R. K.; BISWAS, N.; GUHA, A.; KUOTSU K. Recent advancement of gelatin nanoparticles in drug and vaccine delivery. Int. J. Biol. Macromol., v. 81, p. 317 $331,2015$.

SHAHIDI, F. Omega-3 fatty acids and marine oils in cardiovascular and general health: A critical overview of controversies and realities. J. Funct. Foods, v. 19, p. 797-800, 2015.

WESKA, R. F.; MOURA, J. M.; BATISTA, L. M.; RIZZI, J.; PINTO L. A. A. Optimization of deacetylation in the production of chitosan from shrimp wastes: Use of response surface methodology. J. Food Eng., v. 80, p. 749-753, 2007. 